\title{
Multi-objectivising Combinatorial Optimisation Problems by means of Elementary Landscape Decompositions
}

\author{
Josu Ceberio \\ josu.ceberio@ehu.eus \\ Department of Computer Science and Artificial Intelligence, University of the Basque \\ Country UPV /EHU, Donostia, 20018, Spain
}

Borja Calvo

borja.calvo@ehu.eus

Department of Computer Science and Artificial Intelligence, University of the Basque Country UPV/EHU, Donostia, 20018, Spain

\begin{abstract}
Alexander Mendiburu
alexander.mendiburu@ehu.eus

Department of Computer Architecture and Technology, University of the Basque

Country UPV /EHU, Donostia, 20018, Spain
\end{abstract}

Jose A. Lozano

ja.lozano@ehu.eus

Department of Computer Science and Artificial Intelligence, University of the Basque

Country UPV/EHU, Donostia, 20018, Spain

Basque Center for Applied Mathematics (BCAM), 48009 Bilbao, Spain

\section{Abstract}

In the last decade, many works in combinatorial optimisation have shown that, due to the advances in multi-objective optimisation, the algorithms from this field could be used for solving single-objective problems as well. In this sense, a number of papers have proposed multi-objectivising single-objective problems in order to use multiobjective algorithms in their optimisation. In this paper, we follow up this idea by presenting a methodology for multi-objectivising combinatorial optimisation problems based on elementary landscape decompositions of their objective function. Under this framework, each of the elementary landscapes obtained from the decomposition is considered as an independent objective function to optimise. In order to illustrate this general methodology, we consider four problems from different domains: the quadratic assignment problem and the linear ordering problem (permutation domain), the 0-1 unconstrained quadratic optimisation problem (binary domain), and the frequency assignment problem (integer domain). We implemented two widely known multi-objective algorithms, NSGA-II and SPEA2, and compared their performance with that of a single-objective GA. The experiments conducted on a large benchmark of instances of the four problems show that the multi-objective algorithms clearly outperform the single-objective approaches. Furthermore, a discussion on the results suggests that the multi-objective space generated by this decomposition enhances the exploration ability, thus permitting NSGA-II and SPEA2 to obtain better results in the majority of the tested instances.

Keywords

Multi-objectivisation, Combinatorial Optimisation, Elementary Landscape Decomposition, Multi-objective Evolutionary Algorithm 


\section{Introduction}

A combinatorial optimisation problem consists of finding an optimal solution of a function,

$$
\begin{aligned}
f: \quad X & \longrightarrow \mathbb{R} \\
x & \longmapsto f(x)
\end{aligned}
$$

such that the search space $X$ is a finite or countable infinite set. Usually $f$ is considered as a single-objective function. However, in many real-world problems, the optimisation process may involve multiple objectives (functions) simultaneously. These problems are known as multi-objective optimisation problems (MOPs). Formally, MOPs can be formulated as (Zhang and Li, 2007):

$$
\text { maximize } F(x)=\left(f_{1}(x), \ldots, f_{m}(x)\right), \quad x \in X
$$

where $F: X \longrightarrow \mathbb{R}^{m}$ consists of $m$ real-valued objective (fitness) functions and $\mathbb{R}^{m}$ denotes the objective space.

During the last few decades, multi-objective evolutionary algorithms (MOEAs), such as Non-Dominated Sorting Genetic Algorithm II (NSGA-II) (Deb et al., 2002), Strength Pareto Evolutionary Algorithm 2 (SPEA2) (Zitzler et al., 2001) or Multi-objective Evolutionary Algorithm based on Decomposition (MOEA/D) (Zhang and Li, 2007), have shown their competitiveness when solving MOPs. Considering the unique ability of this type of algorithms to enhance the diversity of the population, the authors have claimed that multi-objective algorithms might be helpful for single-objective optimisation as well (Abbass and Deb, 2003). In fact, according to a recent survey on MOEAs for single-objective optimisation (Segura et al., 2013), a number of papers (Knowles et al., 2001; Scharnow et al., 2005; Neumann and Wegener, 2006) have proposed transforming single-objective problems into MOPs by modifying their fitness function. This procedure, known as multi-objectivisation, was used for the first time by Knowles et al. (2001). The authors distinguished between two types of schemes: decomposition and aggregation. Under the first scheme, the original function $f$ can be decomposed by defining new functions on the solution $x$ (or on subsets of parameters of $x$ ). The second scheme, instead, considers some additional objectives that are used in combination with the original function $f$. In both cases, the multi-objectivisation scheme should guarantee that the optimal solutions in the original problem are in the Pareto set in the multiobjectivised version. Papers on this topic (Knowles et al., 2001; Handl et al., 2008) have demonstrated, for local search algorithms, that the multi-objectivisation techniques are able to find monotonically increasing paths towards global optimal solutions that are not available under the original single-objective problem. Unfortunately, prior to this paper, a general methodology to multi-objectivise efficiently combinatorial optimisation problems did not exist, and therefore, in each case, practitioners had to develop ad-hoc solutions. To this end, Knowles et al. (2001) pointed out that the objectives defined in the new space, need to be as independent as possible.

Following up this idea, in this paper, we extend the work in Ceberio et al. (2015a) and present a general methodology to multi-objectivise single-objective problems based on the decomposition of the original objective function. Particularly, we propose using elementary landscape decomposition techniques in order to decompose the objective function $f$ as a sum of a set of elementary landscapes (functions).

In landscape theory, elementary landscapes (Stadler, 1996) are a class of landscapes whose main characteristic is that they can be modelled using the Grover's wave equation (Grover, 1992) (see Eq. (1) at Section 2). Among its multiple properties, it is possible 
to compute the average value of the objective function in the neighbourhood of a solution using a closed form expression. Moreover, it also allows one to compute landscape ruggedness measures such as the autocorrelation coefficient with a closed form expression (Angel and Zissimopoulos, 2000, 2001; Chicano et al., 2012).

In most of the combinatorial optimisation problems, we do not know a neighbourhood system that is able to produce an elementary landscape. However, Stadler (1996) showed that, for any fitness landscape, if the neighbourhood system holds certain symmetry and regularity properties, then it is possible to decompose $f$ as a sum of elementary landscapes and, thus, express $f$ as

$$
f(x)=\sum_{i=1}^{m} f_{i}(x)
$$

where $m$ is the number of landscapes in the decomposition, and $f_{i}$ is the function related to the $i$-th landscape. For instance, works on elementary landscape decomposition have shown that the landscape associated to the MAX- $k$-SAT under the Hamming neighbourhood can be decomposed as a sum of $k$ elementary landscapes (Sutton et al., 2009), and have proved that the subset sum problem under the same neighbourhood can be decomposed as a sum of two elementary landscapes (Chicano et al., 2011b).

In order to illustrate the multi-objectivisation scheme proposed in this paper and show its general applicability, we choose an elementary landscape decomposition of three problems from different domains: the General Quadratic Assignment Problem (QAP) (Chicano et al., 2011b) (permutation problem), the 0-1 Unconstrained Quadratic Optimisation (UQO) (Chicano and Alba, 2013) (binary problem), and the Frequency Assignment Problem (FAP) (Chicano et al., 2011c) (integer sequence of limited cardinality). For the first decomposition, we distinguish two problems as cases of study for which the decomposition is valid: the classical QAP and the Linear Ordering Problem (LOP) (Martí and Reinelt, 2011; Ceberio et al., 2014b).

According to Chicano et al. (2011b), under the interchange neighbourhood, the objective function of the general QAP can be defined as the sum of, at most, three elementary landscapes. Nevertheless, when translating this decomposition to the particular context of each instance, depending on its characteristics, the decomposition can be reduced as some components turn out to be constant. As a result, the classical QAP and LOP can be reformulated as a two or three-objective problem, one objective for each non-constant elementary landscape. Similarly, UQO and FAP can be reformulated as two-objective problems when considering the respective elementary landscape decompositions under the Hamming neighbourhood.

In general, an arbitrary landscape is not elementary. However, there are a few landscapes, such as that produced by the symmetric traveling salesman problem under the interchange neighbourhood ${ }^{1}$, that are elementary (Whitley et al., 2008). In these cases, the proposed multi-objectivisation scheme is not applicable, however, it must be noted that this does not occur for the majority of the landscapes.

For the sake of demonstrating the validity of the proposed methodology, we ran two MOEAs, NSGA-II and SPEA2, on the four multi-objectivised problems, and compared their performances with that of a single-objective GA (SGA) on the native, singleobjective, problems. The experiments conducted on large benchmarks of instances confirmed that the multi-objective approaches are preferred to the single-objective

\footnotetext{
${ }^{1}$ The interchange neighbourhood considers that two solutions (permutations) are neighbours if one is obtained by interchanging two elements in the other.
} 
approach. A posterior analysis of the experimental results suggests that the multiobjective space defined by the elementary landscape decomposition provides a framework that permits NSGA-II and SPEA2 to diversify the search and, in almost all the cases, to obtain better global results.

The remainder of the paper is organised as follows: in Section 2, the theory on elementary landscape decomposition is introduced. With illustrative purposes, the decomposition for the general formulation of the QAP is also summarised. Section 3 is devoted to describing the multi-objectivisation proposed for each of the problems: QAP, LOP, UQO and FAP. Next, Section 4 introduces a broad experimental study on the four problems. Then, in order to explain the success of the proposed methodology, a discussion is introduced in Section 5. Finally, general conclusions and ideas for future work are presented in Section 6.

\section{Elementary Landscape Decomposition}

In combinatorial optimisation, a fitness landscape is a triple $(X, N, f)$ where $X$ denotes the search space of solutions, $f: X \rightarrow \mathbb{R}$ defines the objective (fitness) function, and the neighbourhood operator $N$ assigns a set of neighbouring solutions $N(x) \in X$ to each solution $x \in X$.

In 1992, Grover (1992) demonstrated that some fitness landscapes arising from certain classes of combinatorial optimisation problems could be modelled using the socalled Grover's wave equation:

$$
\underset{y \in N(x)}{\operatorname{avg}}\{f(y)\}=f(x)+\frac{k}{|N(x)|}(\bar{f}-f(x))
$$

where $\bar{f}$ denotes the average fitness value of all the solutions in the search space, $|N(x)|$ is the size of the neighbourhood, and $k$ is a constant value. Furthermore, Grover proved that, if a landscape satisfies Eq. (1), then all the local maxima solutions are greater than $\bar{f}$ and all the local minima are lower than $\bar{f}$. This class of landscapes was called elementary landscapes by Stadler (2002). According to Stadler, being $\Delta$ the Laplacian matrix associated to $X$ and $N$, a landscape $(X, N, f)$ is elementary if the function $f$ is an eigenvector of $\Delta$ with an eigenvalue $\lambda>0$.

In general, an arbitrary landscape is not elementary, however, Stadler (1996) showed that any landscape can be decomposed as a sum of elementary landscapes if the neighbourhood system considered is regular $(|N(x)|=d>0$, for all $x \in X)$ and symmetric (for all $x, y \in X, y \in N(x) \Longleftrightarrow x \in N(y)$ ). As the author stated, we know that, if a square matrix $Q$ (with real entries) of size $|X|$ is symmetric, then there exists an orthogonal basis in the vector space $\mathbb{R}^{|X|}$ which is composed of eigenvectors of $Q$. Thus, every vector of $\mathbb{R}^{|X|}$ can be written as the weighted sum of the vectors in the orthogonal basis. Since the Laplacian matrix $\Delta$ is a symmetric square matrix with real entries, then it can be deduced that there exists an orthogonal basis of eigenvectors associated to it. As a consequence, $f$ can be decomposed as the weighted sum of a set of elementary functions.

For instance, Rockmore et al. (2002) showed that the landscape produced by the general formulation of the QAP (see Eq. (4)) under the interchange neighbourhood can be written as a sum of three elementary landscapes and, later, Chicano et al. (2011b) gave the exact expression of this decomposition. Since this point is an essential part of this work, in what follows, we provide a general overview of the decomposition for the QAP summarised from Chicano et al. (2011b) (which is also valid for the LOP). As regards the UQO and FAP, for the sake of brevity, the respective decompositions have 
been omitted, and we recommend the interested readers to address the original works by Chicano and Alba (2013) and Chicano et al. (2011c).

\subsection{Elementary Landscape Decomposition for the Quadratic Assignment Problem}

The Quadratic Assignment Problem (QAP) (Koopmans and Beckmann, 1955) is the problem of allocating a set of facilities to a set of locations, with a cost function associated to the distance and flow between the facilities. The objective is to assign each facility to a location, such that the total cost is minimised. Specifically, we are given two real-valued matrices $\mathbf{D}=\left[d_{i, j}\right]_{n \times n}$ and $\mathbf{H}=\left[h_{k, l}\right]_{n \times n}$, where $d_{i, j}$ stands for the distance between location $i$ and location $j$, and $h_{k, l}$ denotes the flow between facility $k$ and facility $l$. Given $n$ facilities, a solution of the QAP is encoded as a permutation $\sigma=(\sigma(1) \ldots \sigma(n))$ where $\sigma(i)(i=1, \ldots, n)$ represents the facility that is allocated to the $i$-th location. The objective (fitness) value of any given permutation is calculated by the following function:

$$
f(\sigma)=\sum_{i=1}^{n} \sum_{j=1}^{n} d_{i, j} h_{\sigma(i), \sigma(j)}
$$

Since the QAP belongs to the class of permutation problems, in the rest of this section, we will adopt the mathematical notation used for the symmetric group. Instead of $x$ and $X$, we will use, from now on, $\sigma$ and $\pi$ to denote solutions, and $\mathbb{S}_{n}$ to denote the search space.

In order to analyse the elementary components of the fitness function of the QAP, it is useful to separate the instance-related part and the problem-related part. Therefore, we start the decomposition procedure by rewriting the fitness function of the QAP as:

$$
f(\sigma)=\sum_{i, j=1}^{n} \sum_{p, q=1}^{n} d_{i, j} h_{p, q} \delta_{\sigma(i)}^{p} \delta_{\sigma(j)}^{q}
$$

where $\delta_{\sigma(i)}^{p}$ denotes the Kronecker's delta and returns 1 if $\sigma(i)=p$, and 0 otherwise. At this point, we extend the decomposition of $f$ to a more general function $g$, in which the instance-related part of Eq. (3), the product $d_{i, j} h_{p, q}$, is replaced with a new variable $\psi_{i j p q}$. Alternatively, the problem-related part $\delta_{\sigma(i)}^{p} \delta_{\sigma(j)}^{q}$, is rewritten as the parameterised function $\varphi_{(i, j)(p, q)}(\sigma)$. Thus, the generalised QAP function is defined as:

$$
g(\sigma)=\sum_{i, j, p, q=1}^{n} \psi_{i j p q} \varphi_{(i, j)(p, q)}(\sigma)
$$

where Eq. (3) is a particular case in which $\psi_{i j p q}=d_{i, j} h_{p, q}$.

No neigbourhood structure that produces an elementary landscape for the function $g$ is known. However, under the interchange neighbourhood (regular and symmetric), there exists an orthogonal basis of functions that permits us to decompose $g$. To this end, Chicano et al. (2011b) focused exclusively on the decomposition of the problemrelated part, since any result on $\varphi_{(i, j)(p, q)}$ can be extended to any linear combination of it, and subsequently to $g$.

First, we distinguish two cases of the function $\varphi$ under the interchange neighbor- 
hood: 1$) i=j \wedge p=q$ and 2) $i \neq j \wedge p \neq q^{2}$. So, we rewrite Eq. (4) as follows:

$$
g(\sigma)=\sum_{i, p=1}^{n} \psi_{i i p p} \varphi_{(i, i)(p, p)}(\sigma)+\sum_{\substack{i, j, p, q=1 \\ i \neq j, p \neq q}}^{n} \psi_{i j p q} \varphi_{(i, j)(p, q)}(\sigma)
$$

When $i=j \wedge p=q$, we see that the function $\varphi$ is elementary and complies with Eq. (1) (with $k=n$ ) by demonstrating that the equation below holds for two constants $a$ and $b$ :

$$
\underset{\pi \in N(\sigma)}{\operatorname{avg}}\{\varphi(\pi)\}=a \varphi(\sigma)+b
$$

Nonetheless, it is not elementary when $i \neq j \wedge p \neq q$. In order to decompose it as a sum of elementary functions, Chicano et al. (2011b) introduced a set of auxiliary functions (see Eq. (8)) that provide the required orthogonal basis in the decomposition. Particularly, the authors prove that the right-hand term $\varphi_{(i, j)(p, q)}$ (see Eq. (5)) can be decomposed as the sum of three elementary functions. Note that, in the QAP (and also in the LOP), the diagonal values of $\mathbf{D}=\left[d_{i, j}\right]_{n \times n}$ and $\mathbf{H}=\left[h_{k, l}\right]_{n \times n}$ are 0 , and, so, the first summing term in Eq. (5) (related to $\psi_{i i p p}$ ) can be discarded. Then, the fitness function of the general QAP, $g$, is finally decomposed as follows:

$$
\begin{aligned}
g(\sigma)= & \sum_{\substack{i, j, p, q=1 \\
i \neq j, p \neq q}}^{n} \psi_{i j p q} \frac{\Omega_{(i, j)(p, q)}^{1}(\sigma)}{2 n} \\
& +\sum_{\substack{i, j, p, q=1 \\
i \neq j, p \neq q}}^{n} \psi_{i j p q} \frac{\Omega_{(i, j)(p, q)}^{2}(\sigma)}{2(n-2)}+\sum_{\substack{i, j, p, q=1 \\
i \neq j, p \neq q}}^{n} \psi_{i j p q} \frac{\Omega_{(i, j)(p, q)}^{3}(\sigma)}{n(n-2)}
\end{aligned}
$$

where $\Omega_{(i, j)(p, q)}^{1}, \Omega_{(i, j)(p, q)}^{2}$ and $\Omega_{(i, j)(p, q)}^{3}$ stand for the elementary functions, and are defined as

$$
\Omega_{(i, j)(p, q)}^{m}(\sigma)= \begin{cases}\alpha & \text { if } \sigma(i)=p \wedge \sigma(j)=q \\ \beta & \text { if } \sigma(i)=q \wedge \sigma(j)=p \\ \gamma & \text { if } \sigma(i)=p \bigoplus \sigma(j)=q \\ \epsilon & \text { if } \sigma(i)=q \bigoplus \sigma(j)=p \\ \zeta & \text { if } \sigma(i) \neq p, q \wedge \sigma(j) \neq p, q\end{cases}
$$

where $1 \leq i, j, p, q \leq n, \alpha, \beta, \gamma, \epsilon, \zeta \in \mathbb{R}$ and $m=1,2,3$. The operator $\bigoplus$ stands for the exclusive OR operation. The sets of parameters for each elementary function are:

\begin{tabular}{c|rrrrr}
$m$ & $\alpha$ & $\beta$ & $\gamma$ & $\epsilon$ & $\zeta$ \\
\hline 1 & $n-3$ & $1-n$ & -2 & 0 & -1 \\
2 & $n-3$ & $n-3$ & 0 & 0 & 1 \\
3 & $2 n-3$ & 1 & $n-2$ & 0 & -1
\end{tabular}

\section{Multi-objectivisation}

As stated in the introduction, in this paper, we propose using elementary landscape decompositions as a general scheme to multi-objectivise single-objective problems. In the following lines, we characterize the solutions in the Pareto Set, and later, we present the multi-objective versions of the problems considered in this paper.

\footnotetext{
${ }^{2}$ Note that $\sigma$ vectors are permutations of size $n$, and, thus, the cases of $\varphi, i=j \wedge p \neq q$ and $i \neq j \wedge p=q$, are zero.
} 
We use $\sigma^{*}$ to denote the global optimal solution for the objective function $f$. We say that a solution $\sigma$ dominates a solution $\sigma^{\prime}$ (denoted as $\sigma \prec y$ ) when there is no objective in the MOP for which $\sigma$ has a worse value than $\sigma^{\prime}$, and there is at least one objective function for which $\sigma$ has a better value than $\sigma^{\prime}$. Otherwise, $\sigma$ and $\sigma^{\prime}$ are non-dominated solutions. The set that contains all non-dominated solutions is called Pareto Set (and is denoted as $P$ ).

So, every global optimal solution $\sigma^{*}$ is in $P$. This is proved by contradiction assuming that $\sigma^{*} \notin P$. Then, there exists $\sigma \in P$ such that $\sigma \prec \sigma^{*}$. From the definition of $\mathrm{P}$, this implies that $f_{i}(\sigma) \leq f_{i}\left(\sigma^{*}\right)$ for $i=1, \ldots, m$ and there exists $j \in\{1, \ldots, m\}$ such that $f_{j}(\sigma)<f_{j}\left(\sigma^{*}\right)$. Thus, $\sum_{i=1}^{m} f_{i}(\sigma)<\sum_{i=1}^{m} f_{i}\left(\sigma^{*}\right)$ which contradicts that $\sigma^{*}$ is a global optima. So $\sigma^{*} \in P$.

Note that, in the case that there exists a unique global optimum solution, then $\forall \sigma \neq \sigma^{*} \in P, \exists i \in\{1, \ldots, m\}$ such that $f_{i}(\sigma)<f_{i}\left(\sigma^{*}\right)$.

In what follows, we present the multi-objectivised forms of the QAP, LOP, UQO and FAP based on the respective elementary landscape decompositions proposed in the literature.

\subsection{The classical Quadratic Assignment Problem}

The decomposition proposed in Eq. (7) for the function $g$ corresponds to the general case of the QAP. Nonetheless, we are interested in the classical definition of the QAP, where $\psi_{i j p q}=d_{i, j} h_{p, q}$. As a result, the classical QAP is multi-objectivised into these three functions, each of them based on one elementary landscape:

$$
\begin{aligned}
f_{1}(\sigma) & =\sum_{\substack{i, j, p, q=1 \\
i \neq j, p \neq q}}^{n} d_{i, j} h_{p, q} \frac{\Omega_{(i, j)(p, q)}^{1}(\sigma)}{2 n} \\
f_{2}(\sigma) & =\sum_{\substack{i, j, p, q=1 \\
i \neq j, p \neq q}}^{n} d_{i, j} h_{p, q} \frac{\Omega_{(i, j)(p, q)}^{2}(\sigma)}{2(n-2)} \\
f_{3}(\sigma) & =\sum_{\substack{i, j, p, q=1 \\
i \neq j, p \neq q}}^{n} d_{i, j} h_{p, q} \frac{\Omega_{(i, j)(p, q)}^{3}(\sigma)}{n(n-2)}
\end{aligned}
$$

It is worth noticing that, when the entries in $\mathbf{D}=\left[d_{i, j}\right]_{n \times n}$ are symmetric with respect to the main diagonal (this occurs in the majority of the instances in the literature), then $f_{1}$ is constant, and, thus, the multi-objective formulation is simplified to two objectives. Specifically, the equality $d_{i, j} h_{p, q}=d_{j, i} h_{p, q}$ permits to group summing terms in $f_{1}$ (by extracting common factors) in three groups under the coefficients $(\alpha+\beta),(\gamma+\epsilon)$ and $2 \zeta$, respectively. Taking into account the parameters of the first elementary function $\Omega^{1}$ $(\alpha=n-3, \beta=1-n, \gamma=-2, \epsilon=0$ and $\zeta=-1)$, i.e, $(\alpha+\beta)=(\gamma+\epsilon)=2 \zeta=-2$, then, any term in the sum is multiplied by the same coefficient, -2 , regardless of $\sigma$. This implies that $f_{1}$ is constant for any $\sigma \in \mathbb{S}_{n}$ whenever the entries in $\mathbf{D}=\left[d_{i, j}\right]_{n \times n}$ are symmetric.

The time complexity of calculating the decomposition of any solution in the search space is in general $O\left(n^{4}\right)$. However, when the matrix $\mathbf{D}=\left[d_{i, j}\right]_{n \times n}$ is symmetric, then the decomposition can be efficiently computed in $O\left(n^{3}\right)$. Note that in the singleobjective case it is $O\left(n^{2}\right)$. 


\subsubsection{The Linear Ordering Problem}

As explained in the introduction, the decomposition of the general QAP is also valid for the Linear Ordering Problem (LOP) (Martí and Reinelt, 2011; Ceberio et al., 2014b) since this problem is a particular case of the previous one. In the LOP, we are given a matrix $\mathbf{B}=\left[b_{k, l}\right]_{n \times n}$ of numerical entries, and the task consists of finding a simultaneous permutation $\sigma$ of the rows and columns of $\mathbf{B}$, such that the sum of the entries above the main diagonal is maximised. The equation below formalises the LOP function:

$$
f(\sigma)=\sum_{i=1}^{n-1} \sum_{j=i+1}^{n} b_{\sigma(i), \sigma(j)}
$$

where $\sigma(i)$ denotes the index of the row (and column) ranked at position $i$ in the solution $\sigma$.

The LOP can be seen as a particular case of the general QAP in which:

- matrix $\mathbf{B}$ corresponds to matrix $\mathbf{H}$.

- the entries in $\mathbf{D}=\left[d_{i, j}\right]_{n \times n}$ above the main diagonal are 1 , and the rest 0 .

- the diagonal values in $\mathbf{D}=\left[d_{i, j}\right]_{n \times n}$ and $\mathbf{H}=\left[h_{k, l}\right]_{n \times n}$ are 0 .

As a result, the LOP is multi-objectivised as

$$
\begin{aligned}
f_{1}(\sigma) & =\sum_{\substack{i, j, p, q=1 \\
i>j, p \neq q}}^{n} b_{p, q} \frac{\Omega_{(i, j)(p, q)}^{1}(\sigma)}{2 n} \\
f_{3}(\sigma) & =\sum_{\substack{i, j, p, q=1 \\
i>j, p \neq q}}^{n} b_{p, q} \frac{\Omega_{(i, j)(p, q)}^{3}(\sigma)}{n(n-2)}
\end{aligned}
$$

As can be appreciated, $f_{2}$ has been omitted from the multi-objectivisation. In this case, due to the description of the matrix $\mathbf{D}$, and taking into account the parameter set assigned to $\Omega^{2}(\alpha=\beta=n-3, \gamma=\epsilon=0$ and $\zeta=1), f_{2}$ turns out constant for any solution $\sigma \in \mathbb{S}_{n}$. A detailed proof of this result can be found in Appendix A. As for the $\mathrm{QAP}$, based on the characteristics of the problems, the decomposition can be efficiently computed in $O\left(n^{3}\right)$, and the single-objective case in $O\left(n^{2}\right)$.

\subsection{The 0-1 Unconstrained Quadratic Optimisation Problem}

In the 0-1 Unconstrained Quadratic Optimisation Problem (from now on UQO), we are given a matrix $\mathbf{Q}=\left[q_{k, l}\right]_{n \times n}$ of numerical entries, and the problem consists of finding the solution $x \in\{0,1\}^{n}$ (a binary vector) that maximizes the following function:

$$
f(x)=\sum_{i=1}^{n} \sum_{j=1}^{n} q_{i, j} x(i) x(j)
$$

According to Chicano and Alba (2013), the fitness landscape produced by UQO under the Hamming neighbourhood can be decomposed as a sum of two subfunctions. As a 
result, the UQO is multi-objectivised as:

$$
\begin{aligned}
& f_{1}(\sigma)=-\frac{1}{4} \sum_{i=1}^{n} v_{i}(1-2 x(i)) \\
& f_{2}(\sigma)=\frac{1}{4} \sum_{\substack{i, j=1 \\
i \neq j}}^{n} q_{i, j}(1-2|x(i)-x(j)|)
\end{aligned}
$$

where $v_{i}=\sum_{j=1}^{n}\left(q_{i, j}+q_{j, i}\right)$. The time complexity of computing the decomposition is equal to the single-objective case, $O\left(n^{2}\right)$.

\subsection{The Frequency Assignment Problem}

The Frequency Assignment Problem (FAP) is the problem of assigning $r$ channels to $n$ transceivers, such that the interference costs derived from the assignment of the channels to transceivers is minimized (Aardal et al., 2007). In general, the interferences are produced between transceivers that are close to each other, and thus, in such cases it is desirable to assign channels that are far from each other in the frequency band. Formally, in the general form of the problem, we are given an array of weights $\mathbf{W}=\left[w_{i, j}^{p, q}\right]_{n \times n \times r \times r}$ of numerical entries, where each element $w_{i, j}^{p, q}$ stands for the cost of assigning channel $p$ to transceiver $i$ and channel $q$ to transceiver $j$ (Chicano et al., 2011a). The problem consists of finding the vector of integers $x$ of size $n$ that minimizes the objective function:

$$
f(x)=\sum_{i=1}^{n} \sum_{j=1}^{n} w_{i, j}^{x(i), x(j)}
$$

As for UQO, the landscape produced under the Hamming neighbourhood can be decomposed as a sum of two subfunctions. Then, the FAP is multi-objectivised as:

$$
\begin{aligned}
& f_{1}(x)=\frac{1}{r} \sum_{\substack{i, j=1 \\
i \neq j}}^{n} \sum_{p, q=1}^{r} w_{i, j}^{p, q} \phi_{i, j, r-2}^{p, q}(x) \\
& f_{2}(x)=-\frac{1}{r} \sum_{\substack{i, j=1 \\
i \neq j}}^{n} \sum_{p, q=1}^{r} w_{i, j}^{p, q} \phi_{i, j,-2}^{p, q}(x)+\sum_{i=1}^{n} \sum_{p=1}^{r} w_{i, i}^{p, p} \delta_{x(i)}^{p}
\end{aligned}
$$

where $\phi_{i, j, \alpha}^{p, q}(x)$ is defined as:

$$
\phi_{i, j, \alpha}^{p, q}(x)=\left\{\begin{array}{cl}
\alpha & \text { if } x(i)=p \wedge x(j)=q \\
-1 & \text { if } x(i)=p \oplus x(j)=q \\
0 & \text { otherwise }
\end{array}\right.
$$

The time complexity of computing the decomposition is $O\left(n^{4}\right)$, while in the singleobjective case it is $O\left(n^{2}\right)$.

\section{Experimental Study}

In the previous section, we presented multi-objectivised versions for the four problems (with two or three objectives, depending on the case). In what follows, in order to evaluate whether optimising in the described multi-objective spaces is preferred 
to optimising the single-objective (native) functions, we compare the performance of NSGA-II (Deb et al., 2002) and SPEA2 (Zitzler et al., 2001) on the multi-objectivised formulations, with a single-objective GA (SGA) that optimises the native versions of the problems.

\subsection{The algorithms: SGA, NSGA-II and SPEA2}

The SGA is the reference algorithm used to compare the multi-objective approaches in this experimental study. Following a standard design, at every generation, SGA evolves a population of candidate solutions of the problem by performing selection, mating and update operations. Particularly, it implements a binary tournament selection algorithm to form the mating pool of solutions. Regarding the crossover operator, in the QAP and LOP, the one proposed by Lim et al. (2000) for permutations has been considered, and the single-point crossover for the UQO and FAP. Similarly, the exchange mutation operator was used for the QAP and LOP, two bit-flip for the UQO, and two transceiver mutation for the FAP. All the operators have been selected without performing previous experiments. Next, the offspring and parent populations are combined in the ensuing iteration population, where only the fittest solutions survive.

The first MOEA described in this work is the Non-Dominated Sorting Algorithm II (NSGA-II) (Deb et al., 2002) which is one of the most referenced algorithms for multiobjective optimisation. Presented as an improvement of NSGA, at every generation $t$, NSGA-II combines the parent and offspring populations in a new population $P_{t}$, and ranks the solutions according to a fast non-dominance sorting algorithm. This algorithm is an iterative scheme that, at each step, finds the set of non-dominated solutions in the population $P_{t}$, also known as the front, and moves them into the next generation population $P_{t+1}$. If the size of the front is smaller than the available space in $P_{t+1}$, all the members of the front are chosen. The remaining members of the new population will be chosen from the subsequent non-dominated fronts. This procedure continues until no more fronts can be accommodated. In general, the last front accommodated will be partially chosen, since, presumably, it will be larger than the empty space in $P_{t+1}$. In order to choose the solutions that will survive from that last front, NSGAII implements the crowded-comparison operator which, based on a density estimation metric called crowding distance, selects the solutions that are spread out in that front.

The second MOEA included is the Strength Pareto Evolutionary Algorithm 2 (SPEA2) (Zitzler et al., 2001). The design of SPEA2 is proposed on the basis of two goals:

- to minimise the distance to the optimal front.

- to maximise the diversity of the generated solutions.

In order to find a trade-off between both goals, SPEA2, in addition to the regular population, uses an external population called archive to store all the non-dominated solutions found so far. Starting with an initial population and an empty archive, SPEA2 iterates according to the following steps. At the beginning, all the non-dominated solutions in the population are copied to the archive, removing any duplicity. If the size of the update exceeds the predefined size of the archive, the most representative solutions of the front are selected by performing a clustering technique. Then, all the solutions in the population and archive are assigned a fitness value as follows:

- every solution $i$ in the archive is assigned a strength value which describes the number of solutions in the population that it dominates or are equal to it in terms 
Multi-objectivising Combinatorial Optimisation Problems by means of ELD

Table 1: Parameter settings for the SGA, NSGA-II and SPEA2.

\begin{tabular}{l|rrrrr} 
Algorithm & Pop. Size & Sel. Size & Off. Size & crossover ratio & mutation ratio \\
\hline SGA & $8 n$ & $8 n$ & $8 n$ & 1.0 & 1.0 \\
NSGA-II & $8 n$ & $8 n$ & $8 n$ & 1.0 & 1.0 \\
SPEA2 & $8 n$ & $4 n$ & $4 n$ & 1.0 & 1.0
\end{tabular}

of objective values, divided by the population size plus one. This strength value is also the fitness value for the solutions in the archive.

- the fitness value of all the solutions in the population is calculated by summing the strength values of all archive members that dominate it.

If the solutions in the current generation do not dominate each other, then very little information is obtained from the dominance relation. In this sense, SPEA2 incorporates a density value to the solutions in the population, which is calculated as the inverse of the distance to the $k$-th nearest neighbours. Afterwards, the mating pool is obtained from the union of the population and the archive by means of binary tournaments. For a more detailed description, the interested reader is referred to Zitzler et al. (2001).

Since it is the aim of this experimental study to evaluate the benefits of optimising in the multi-objective space rather than in the single-objective space, the same crossover and mutation operators as in SGA were implemented for NSGA-II and SPEA2.

\subsection{Settings}

In Table 1, the population, selection and offspring sizes for the three algorithms are detailed. These parameters have been set taking into account the recommendations in the original works and some preliminary experiments.

With respect to the stopping criterion, a number of solution evaluations have been set: for the QAP and LOP each of the algorithms performs $1000 n^{2}$ solution evaluations, and $100 n^{2}$ in the case of the UQO and FAP .

The three algorithms, NSGA-II, SPEA2 and SGA, have been implemented in C++, using, in some cases, parts of the original source codes of the algorithms downloaded from the web-pages of the authors ${ }^{4}$. The experimentation was performed on a cluster of 20 nodes, each of them equipped with two Intel Xeon X5650 CPUs and 48GB of memory.

\subsection{Experimental Benchmarks}

The experimental framework proposed in this work consists of the following benchmarks:

- 288 QAP instances selected from different sources: 188 instances have been obtained from the QAP Library (Burkard et al., 1997), and 100 extra instances from the Taixxeyy benchmark (Drezner et al., 2005). The size of the instances ranges from 5 to 175 .

- For the LOP, we used 176 instances downloaded from the LOLIB benchmark. In this case, the size of the instances ranges between 44 and 163.

\footnotetext{
${ }^{3}$ Due to the limited computation capabilities available and the size of FAP and UQO instances, a lower number of solutions evaluations have been considered.

${ }^{4}$ NSGA-II: http://www.egr.msu.edu/ kdeb/codes.shtml and SPEA2: http://www.tik.ee. ethz.ch/sop/pisa/selectors/spea2/?page=spea2 $\cdot$ php 
- As regards the UQO, 50 instances proposed in Beasley (1998) and 45 instances used in Glover et al. (1998) have been collected. The size of the instances ranges between 50 and 1000.

- Finally, we generated 45 instances for the FAP. The number of transceivers considered are 25, 50 and 75 (with a different set of frequencies to allocate in each case). These instances have been generated as follows:

- the $w_{i, j}^{p, q}$ parameters that make up instances have been sampled uniformly at random from the range $\left[n, n^{2}\right]$, and then weighted with the distance factor between frequencies $\frac{1}{|p-q|+1}$.

- following a realistic scenario, the instances hold the equality $w_{i, j}^{p, q}=w_{i, j}^{q, p}=$ $w_{j, i}^{p, q}=w_{j, i}^{q, p}$.

\subsection{Experiments \& Results}

Each algorithm - instance pair was run 30 times, and the average values of the best fitness function obtained in each run were computed for comparison. In Table 2, we show for each multi-objective approach the number of instances on each benchmark for which the obtained average values outperformed those of SGA (see Avg. columns). Alternatively, we also computed the number of instances for which SGA was the best performing algorithm (see SGA column).

In order to assess whether the differences observed are statistically significant, we performed a statistical test on the results obtained by each pair of algorithms (SPEA2SGA and NSGA-II-SGA) on each instance, i.e., the data for each test is built up with 60 objective values (30 repetitions $\times 2$ algorithms). As the distribution of the results does not fit a Gaussian distribution, we have used non-parametric tests to perform the comparison. Moreover, due to the nature of the results, it is quite frequent to have repetitions in the samples. This means that ranking-based tests, which assume the continuity of the results (i.e., that there are no repetitions in the samples), are not appropriate. For these reasons, we apply a permutation test (Good, 2013) whose assumption is that, under the null hypothesis, the results of the compared algorithms are exchangeable. To this end, we computed the average values of the results obtained by each algorithm and calculated the absolute difference $D_{0}$ between them. Then, we shuffled the $60 \mathrm{ob}-$ jective values between the two algorithms 100000 times, and calculated the absolute difference between average values in each case. Finally, the $p$-value was calculated as the proportion of times for which the difference of average values was greater than $D_{0}{ }^{5}$. A level of significance $\alpha=0.05$ has been set in all cases. Since the analysis corresponds to a multiple hypothesis testing scenario (one per instance), the $p$-values have been corrected with the method of Finner (1999). From this analysis, we counted the number of instances for which NSGA-II and SPEA2 perform significantly better than SGA in each benchmark. The results have been summarised in Table 2, Stat columns.

According to the average values obtained in the QAP, NSGA-II was better than SGA in 224/265 instances (84.5\%), SPEA2 in 241/265 (90.9\%), and the single-objective option (SGA) was better than the multi-objective approaches in 15/265 (5.2\%) ${ }^{6}$. Note that, in 23 instances (values within parenthesis), the three algorithms obtained equal

\footnotetext{
${ }^{5}$ The statistical tests in this work have been carried out with the scmamp package for $\mathrm{R}$ (Calvo and Santafe, 2016), and following the guidelines included in the documentation of the package.

${ }^{6}$ Instances, source-codes and raw results can be downloaded from https://github.com/sgpceurj/ ECJ_Multiobjectivisation.git.
} 
Table 2: Summary of the number of instances for which NSGA-II and SPEA2 outperform SGA and vice versa. Values in Avg. and Stat columns correspond to the comparisons with average fitness values obtained throughout 30 repetitions, and statistical tests respectively. The numbers within parenthesis denote the number of instances in which the three algorithms reached the best known result. Such cases were discarded from the analysis since they are not useful for comparison purposes.

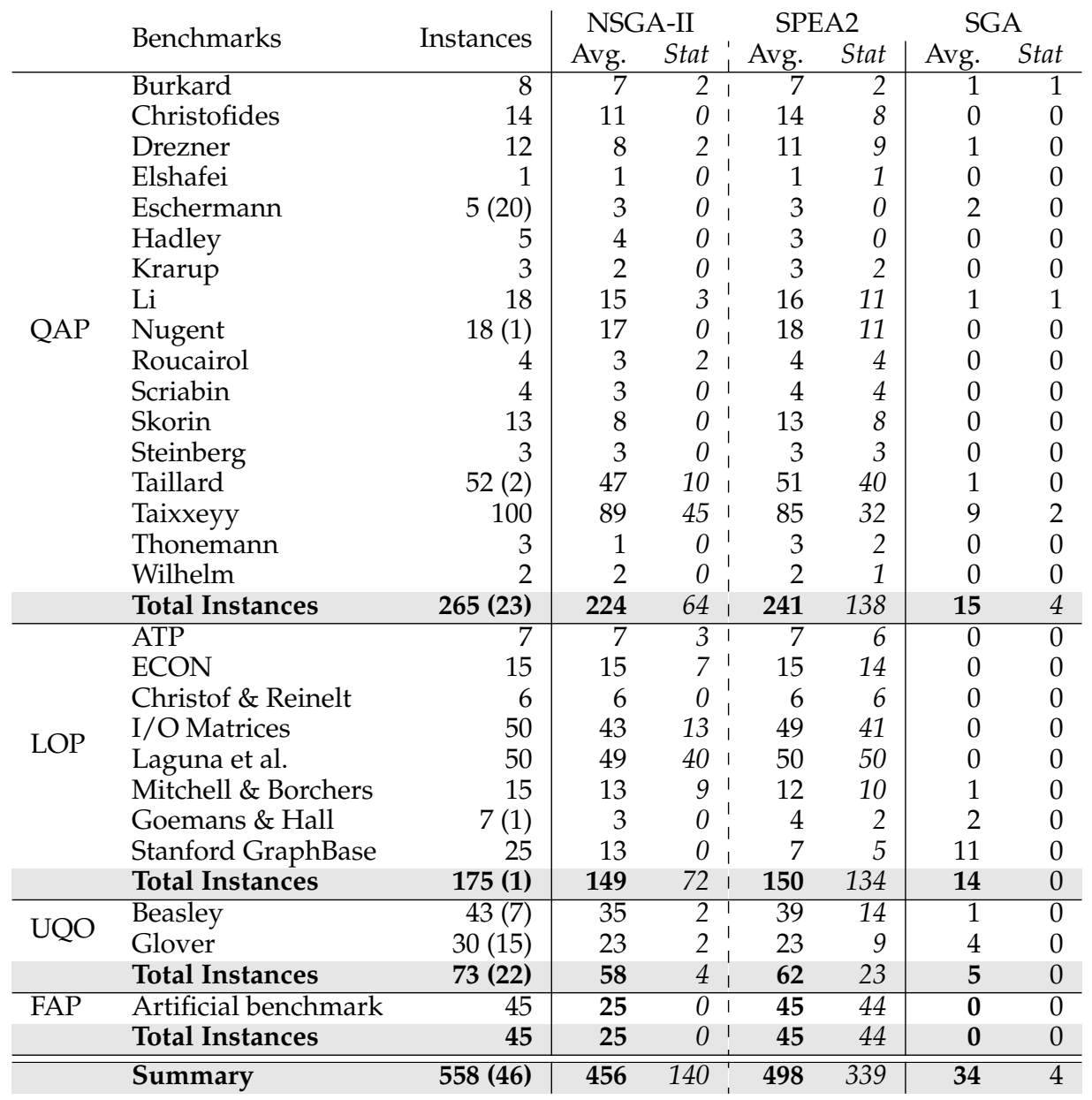


solutions. Taking into account that such cases are not useful to compare the performance of the algorithms, they have not been considered hereinafter. According to the statistical analysis, NSGA-II performs significantly better than SGA in 64/265 instances (24.1\%), and SPEA2 in 144/265 (54.3\%).

The conducted experiments on the LOP point out that NSGA-II obtained better results than SGA in 149 instances out of 175 (85.1\%), SPEA2 in 150/175 (85.7\%), and SGA is better than both algorithms in 14/175 (8\%). According to the statistical analysis, NSGA-II performs significantly better than SGA in 72/175 (41.1\%) instances and SPEA2 in $134 / 175(76.5 \%)$ instances.

As regards the UQO and FAP problems, a similar trend can be observed in the results. In the UQO, NSGA-II and SPEA2 beat SGA in 58/73 (79.4\%) and 62/73 (84.9\%) respectively, while SGA was the preferred approach in 5/73 (6.8\%). In the FAP, NSGAII did not show such competitive behaviour as it beat SGA only in 25 out of 45 instances (55.5\%). However, SPEA2 obtained better results than SGA in 44 instances out of 45 . Finally, according to the statistical analysis, NSGA-II did perform significantly better than SGA in 4/53 of the UQO instances, and in 0 instances of the FAP. SPEA2 outperformed SGA in 23/73 (31.5\%) and 44/45 (97.7\%) instances respectively.

In summary, we observed that NSGA-II and SPEA2 obtained better average results than SGA in $81.7 \%$ and $89.2 \%$ of the instances respectively, while SGA was preferred to multi-objective approaches in $6.0 \%$. The statistical analysis confirmed those results, with SGA being significantly preferred to either NSGA-II or SPEA2 in only in 4 comparisons (among all the pairwise comparisons).

In order to illustrate the differences among the algorithms, and also with respect to the best known solutions, in Fig.1, we present, as scatter plots, the best fitness results of each run obtained by the three algorithms on three instances of each problem ${ }^{7}$. Particularly, the relative deviations to the best known values obtained across 30 repetitions have been computed.

The plots show that, on the one hand, the variance of SGA is systematically larger than that of NSGA-II and SPEA2. Additionally, it can be seen that multi-objective algorithms are able to obtain lower deviations to the best known solutions, which demonstrates a better performance of these algorithms. It is worth mentioning that all the deviations reported in Fig. 1 are relatively small as they are below 0.1 .

\section{Discussion}

The experimental study above supported the validity of using elementary landscape decomposition as a general method to multi-objectivise single-objective problems successfully. In fact, NSGA-II and SPEA2 outperformed SGA in $81.7 \%$ and $89.2 \%$ of the instances respectively. The observed result poses the following question: why does the decomposition in elementary landscapes provide a suitable framework to multiobjectivise problems?

It is a question which is difficult to answer, however, there are two characteristics in the methodology presented in the work that, in our opinion, are involved in the success of the proposed multi-objectivisation: 1) the suitability of the elementary landscape decomposition, and 2) the diversification of the search.

As noted in the introduction, according to Knowles et al. (2001), the success of multi-objectivising single-objective problems lies in the intuition that the multiobjective space opens up monotonically increasing paths to the global optimum that

\footnotetext{
${ }^{7}$ The plots of the remaining instances can be found in the online repository as supplementary material
} 


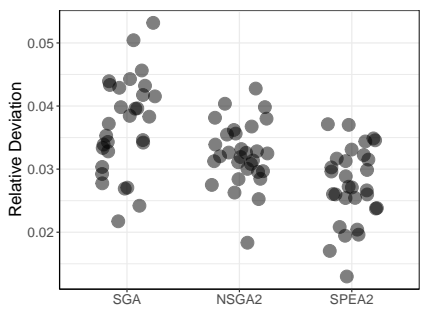

(a) QAP - tai40a

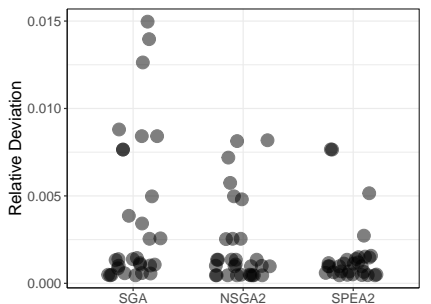

(d) LOP - N-t70d11xx

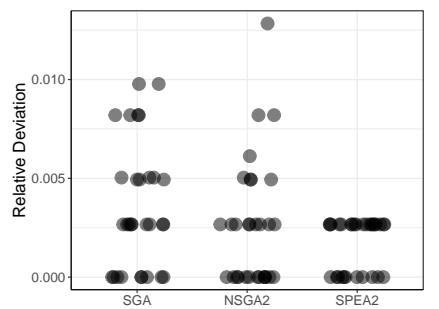

(g) UQO - beas100_7

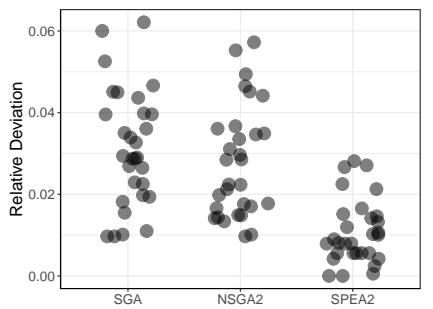

(j) FAP - cebe25_10_2

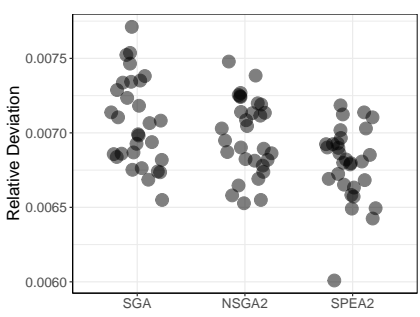

(b) QAP - lipa90a

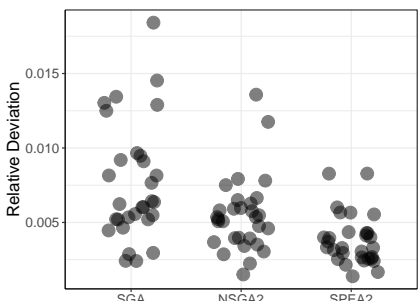

(e) LOP - N-econ77

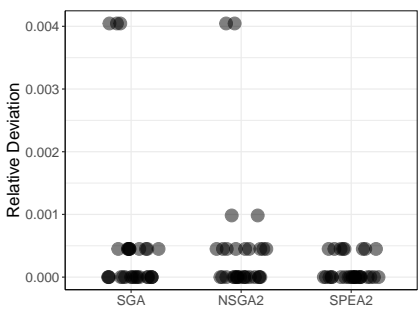

(h) UQO - glov200_4

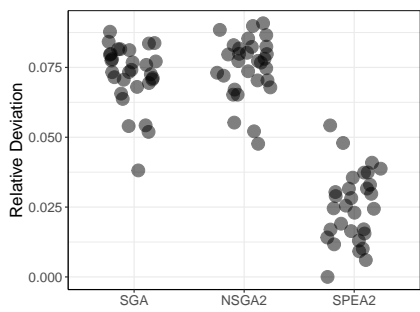

(k) FAP - cebe50_45_2

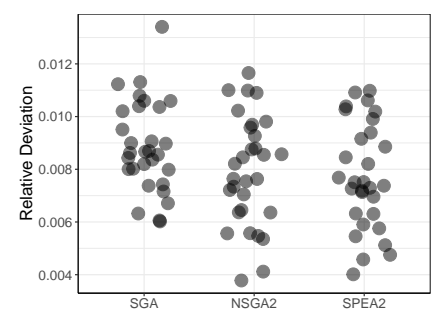

(c) QAP - Wil100

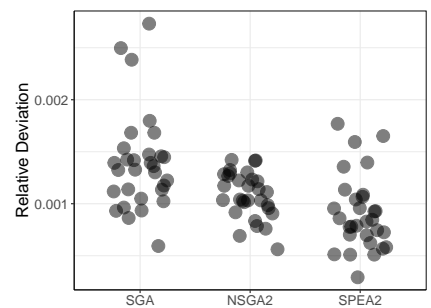

(f) LOP - N-r150d1

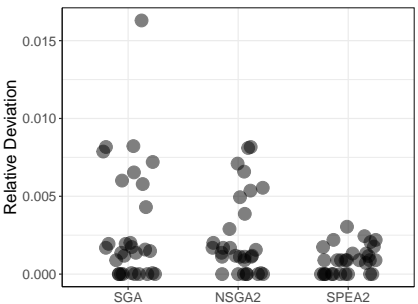

(i) UQO - beas500_5

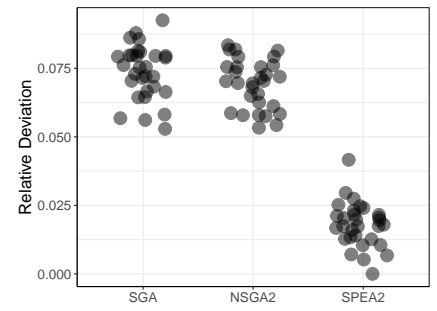

(1) FAP - cebe75_60_1

Figure 1: Scatter plots of the results obtained in three instances for each problem. In each plot, the best results obtained by NSGA-II, SPEA2 and SGA across 30 repetitions are illustrated. Particularly, the normalized deviations of the results obtained with respect to the best known solutions are illustrated. The lower the deviation, the better.

are not available under the original space ${ }^{8}$. Nevertheless, in order to develop successful multi-objective isolations, although no guidelines are available, some general hints

\footnotetext{
${ }^{8}$ The work by Knowles et al. (2001) is focused on local search algorithms. Nonetheless, correlations on the performance of local search algorithms and population-based EAs when using genetic operators that are somehow related with the neighborhoods systems have been reported frequently in the literature (see for instance, Ceberio et al. (2014a)).
} 
are given. In general, most of the decompositions proposed for multi-objectivisation are ad-hoc for each problem, and consider using expertise on the problem. However, Knowles et al. (2001) noted that it is essential to separate out the conflicting aspects of the problem, i.e., to find objectives that are as independent as possible. This way, the different solutions on the Pareto Front correspond to solutions that are more or less good for different objectives. At this point, the proposed decomposition fits with the idea suggested by Knowles et al. (2001), as the elementary landscape decomposition of a given landscape $(X, f, N)$ is the weighted sum of elementary functions (eigenvectors) of the Laplacian matrix $\Delta$ that describes the neighborhood structure $(X, N)$, and the eigenvectors that correspond to the same orthogonal basis associated to $\Delta$.

Another interesting aspect to discuss is that of diversity. In single-objective optimisation, there is one objective value to consider and use as a reference. However, once the problem is efficiently multi-objectivised, the solutions are selected considering multiple objective values, which intrinsically diversifies the search as it enables the selection of solutions that are competitive for different objectives. Additionally, MOEAs incorporate schemes to diversify solutions regarding their genotypic characteristics (and not objective values). Plots in Fig. 2 illustrate the solutions found throughout the optimisation process of a specific QAP (tai40b) instance by SGA, NSGA-II and SPEA2. $x$ - and $y$-axis correspond to the two objectives into which the QAP is multi-objectivised ( $f_{1}$ is constant). As can be seen, the multi-objective approaches clearly diversify the search compared with the single-objective case. This behavior can be extended to the other problems and instances considered in this paper.

\section{Conclusions and Future Work}

In this paper, we presented a general methodology to multi-objectivise single-objective problems based on an elementary landscape decomposition of the fitness function. In order to illustrate this procedure, we considered the decomposition of four problems from different domains, and, based on the analysis of the elementary functions, we transformed the classical QAP, LOP, UQO and FAP into multi-objective problems. In order to study the validity of the proposed multi-objectivisation scheme, we compared the performance of NSGA-II and SPEA2 on the multi-objective problems, with a genetic algorithm, SGA, for the single-objective version.

Experiments on a large set of instances showed that SPEA2 and NSGA-II outperform SGA for a large majority of the evaluated instances. In fact, the statistical test concluded that SPEA2 is the best algorithm followed by NSGA-II, SGA being the least competitive approach.

The methodology presented here could also be applied to other problems for which a decomposition is already known (see Table 3). Note that the listed decompositions are applicable to a larger number of problems. For instance, the decomposition proposed for 0-1 Unconstrained Quadratic Optimisation Problem is equally applicable to special cases of this problem, such as maximum clique, maximum cut, maximum independent set, and many others (Glover et al., 2002).

As this work has been the first attempt to multi-objectivise combinatorial problems by means of elementary landscape decompositions, there are many research issues, both theoretical and practical, that could be investigated in future works:

- A first issue is the choice of the neighbourhood to carry out the elementary landscape decomposition. For instance, in the literature related to the QAP it has been reported that the interchange neighbourhood performs for local search algorithms 


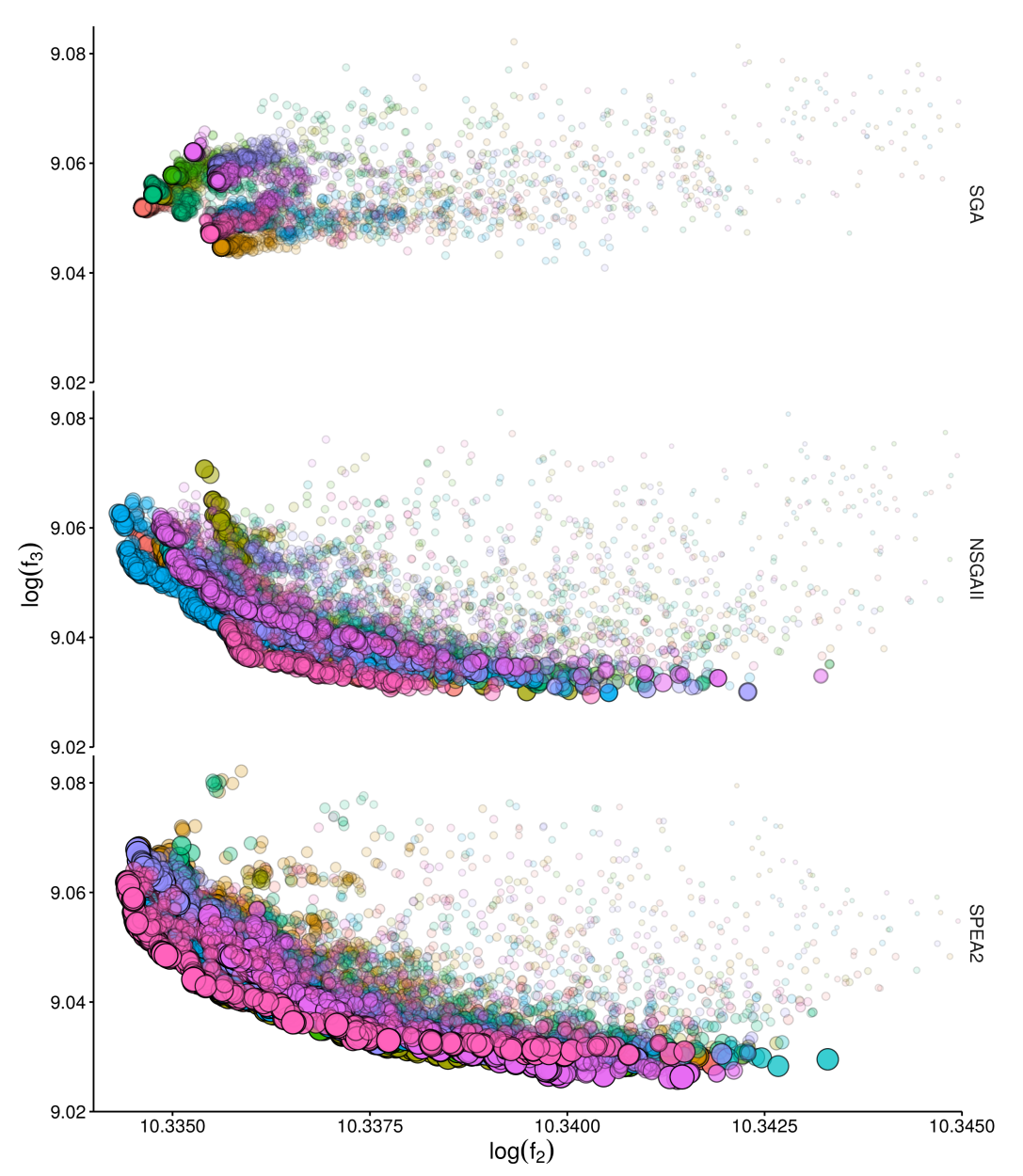

Figure 2: All the solutions observed in the execution of 10 repetitions of SGA, NSGA-II and SPEA 2 on tai40b. The circle size indicates the generation at which the solution was observed (the later the generation, the larger the circle). Each run is presented using a different colour.

the best. So, is the elementary landscape decomposition based on that neighbourhood the most appropriate multi-objectivisation of that problem? Intuition suggests that it is. The literature presents a number of problems for which an elementary landscape decomposition has been reported (see Table 3), unfortunately, we did not find two decompositions for the same problem to be able to answer that question.

In the same line, in the case of the LOP, a large number of papers in the literature have reported that the insert neighbourhood is the most appropriate neighbourhood to use in local search algorithms (Ceberio et al., 2014b). However, in this paper, we used the interchange neighbourhood. Thus, would the decomposition based on the insert neighbourhood provide a better multi-objectivisation than the one used in this work? In this sense, developing an elementary landscape decomposition for the LOP based on the insert neighbourhood is an interesting line for 
Table 3: Relation of elementary landscape decompositions reported in the literature. $\left(^{*}\right)$ The elementary landscape decomposition of the LOP to two components is introduced in this paper.

\begin{tabular}{lcll} 
Problem & Neighborhood & Comp. & Reference \\
\hline \hline Quadratic Assignment & interchange & 3 & Chicano et al. (2010) \\
Linear Ordering & interchange & 2 & $\left({ }^{*}\right)$ \\
DNA Fragment Assembly & interchange & 3 & Chicano et al. (2010) \\
\hline Subset Sum & bit-flip & 2 & Chicano et al. (2011b) \\
Max $k$-sat & bit-flip & $k$ & Rana et al. (1998) \\
Test Suite Minimization & bit-flip & $n+1$ & Chicano et al. (2011a) \\
NK-landscapes & bit-flip & $k+1$ & Sutton et al. (2009) \\
0-1 Unconstrained Quadratic Opt. & bit-flip & 2 & Chicano and Alba (2013) \\
\hline General Frequency Assignment & Hamming & 2 & Chicano et al. (2011c)
\end{tabular}

future research.

- Elaborating the previous questions, should the multi-objective optimisation algorithm consider operators (mutation, crossover...) that are in correspondence with the neighbourhood used in the decomposition (Ceberio et al., 2015b)? This requires extensive research work that is beyond the scope of this paper.

- Results in Fig. 1 showed that the differences with respect to the best known solutions are relatively small, and therefore, another research line for future work could be using the approach proposed in this work as a basis to develop algorithms capable of beating state-of-the-art algorithms.

\section{Appendix A: Proof of simplification of $f_{2}$ in the LOP}

As commented previously in Section 3.1.1, the LOP can be seen as a particular case of the general QAP in which, (i) matrix $\mathbf{B}$ corresponds to matrix $\mathbf{H}$, (ii) the entries in $\mathbf{D}$ above the main diagonal are 1, and the rest 0, (iii) and diagonal values in $\mathbf{D}$ and in $\mathbf{H}$ are 0 . Due to such restrictions, the multi-objectivisation of the LOP is simplified. In the following lines, we prove that $f_{2}$ is constant for any $\sigma \in \mathbb{S}_{n}$.

The $f_{2}$ function can be expressed as follows:

$$
f_{2}(\sigma)=\sum_{\substack{i, j, p, q=1 \\ i \neq j, p \neq q}}^{n} d_{i, j} b_{p, q} \frac{\Omega_{(i, j)(p, q)}^{2}(\sigma)}{2(n-2)}
$$

is simplified due to the values in $\mathbf{D}$, as

$$
f_{2}(\sigma)=\sum_{\substack{i, j, p, q=1 \\ i>j, p \neq q}}^{n} b_{p, q} \frac{\Omega_{(i, j)(p, q)}^{2}(\sigma)}{2(n-2)}=\sum_{i>j}^{n} \sum_{\substack{p, q=1 \\ p \neq q}}^{n} b_{p, q} \frac{\Omega_{(i, j)(p, q)}^{2}(\sigma)}{2(n-2)}
$$

For each pair $(i, j)$ that holds $i>j$, there are $n(n-1)$ coefficients $\Omega_{(i, j)(p, q)}^{2}(\sigma)$, from which there is one $\alpha$ case, one $\beta, 2(n-2) \gamma, 2(n-2) \epsilon$ and $(n-2)(n-3) \zeta$ (see Eq. 8), with values $\alpha=\beta=(n-3), \gamma=\epsilon=0$ and $\zeta=1$. Since $\gamma$ and $\epsilon$ equal to 0 , so $f_{2}(\sigma)$ can 
be rewritten as follows:

$$
f_{2}(\sigma)=\frac{1}{2(n-2)} \sum_{i>j}^{n}\left((n-3)\left(b_{\sigma(i), \sigma(j)}+b_{\sigma(j), \sigma(i)}\right)+\sum_{p, q \neq\{\sigma(i), \sigma(j)\}}^{n} b_{p, q}\right)
$$

And, with some algebra

$$
f_{2}(\sigma)=\frac{1}{2(n-2)}\left((n-3) \sum_{i, j=1}^{n} b_{i, j}+\frac{(n-2)(n-3)}{2} \sum_{i, j=1}^{n} b_{i, j}\right)
$$

, thus, $f_{2}$ is finally the constant value:

$$
f_{2}(\sigma)=\frac{n(n-3)}{4(n-2)} \sum_{i, j=1}^{n} b_{i, j}
$$

\section{Acknowledgements}

This work has been partially supported by the Research Groups 2013-2018 (IT-60913) programs (Basque Government) and TIN2016-78365R (Spanish Ministry of Economy, Industry and Competitiveness). Jose A. Lozano is also supported by BERC 20142017 and Elkartek programs (Basque government) and Severo Ochoa Program SEV2013-0323 (Spanish Ministry of Economy and Competitiveness). Finally, we gratefully acknowledge the recommendations and comments given by Dr. Francisco Chicano, which most certainly enriched this work.

\section{References}

Aardal, K. I., Van Hoesel, S. P., Koster, A. M., Mannino, C., and Sassano, A. (2007). Models and solution techniques for frequency assignment problems. Annals of Operations Research, 153(1):79-129.

Abbass, H. A. and Deb, K. (2003). Searching under multi-evolutionary pressures. In Proceedings of the 4th Conference on Evolutionary Multi-Criterion Optimization, volume 2632 of Lecture Notes in Computer Science, pages 391-404. Springer Berlin Heidelberg.

Angel, E. and Zissimopoulos, V. (2000). On the classification of NP-complete problems in terms of their correlation coefficient. Discrete Applied Mathematics, 99(1-3):261 277.

Angel, E. and Zissimopoulos, V. (2001). On the landscape ruggedness of the quadratic assignment problem. Theoretical Computer Science, 263(1-2):159 - 172. Combinatorics and Computer Science.

Beasley, J. E. (1998). Heuristic algorithms for the unconstrained binary quadratic programming problem. London, UK: Management School, Imperial College, 4.

Burkard, R. E., Karisch, S. E., and Rendl, F. (1997). QAPLIB - a quadratic assignment problem library. Journal of Global Optimization, 10(4):391-403.

Calvo, B. and Santafe, G. (2016). scmamp: Statistical Comparison of Multiple Algorithms in Multiple Problems. The R Journal. R package version 2.0. 
Ceberio, J., Calvo, B., Mendiburu, A., and Lozano, J. A. (2015a). Advances in Artificial Intelligence: 16th Conference of the Spanish Association for Artificial Intelligence, CAEPIA 2015 Albacete, Spain, November 9-12, 2015 Proceedings, volume 9422 of Lectures Notes in Computer Science, chapter Multi-objectivising the Quadratic Assignment Problem by Means of an Elementary Landscape Decomposition, pages 289-300. Springer International Publishing.

Ceberio, J., Irurozki, E., Mendiburu, A., and Lozano, J. A. (2014a). Extending distancebased ranking models in estimation of distribution algorithms. In Proceedings of the 2014 IEEE Congress on Evolutionary Computation, pages 2459-2466. IEEE.

Ceberio, J., Irurozki, E., Mendiburu, A., and Lozano, J. A. (2015b). A review of distances for the Mallows and Generalized Mallows estimation of distribution algorithms. Computational Optimization and Applications, 62(2):545-564.

Ceberio, J., Mendiburu, A., and Lozano, J. A. (2014b). The Linear Ordering Problem Revisited. European Journal of Operational Research, 241(3):686-696.

Chicano, F. and Alba, E. (2013). Elementary landscape decomposition of the 0-1 unconstrained quadratic optimization. Journal of Heuristics, 19(4):711-728.

Chicano, F., Ferrer, J., and Alba, E. (2011a). Elementary landscape decomposition of the test suite minimization problem. In International Symposium on Search Based Software Engineering, pages 48-63. Springer.

Chicano, F., Luque, G., and Alba, E. (2010). Elementary landscape decomposition of the quadratic assignment problem. In Proceedings of the 12th Annual Conference on Genetic and Evolutionary Computation, GECCO '10, pages 1425-1432.

Chicano, F., Luque, G., and Alba, E. (2012). Autocorrelation measures for the quadratic assignment problem. Applied Mathematical Letters, 25(4):698-705.

Chicano, F., Whitley, L. D., and Alba, E. (2011b). A methodology to find the elementary landscape decomposition of combinatorial optimization problems. Evolutionary Computation, 19(4):597-637.

Chicano, F., Whitley, L. D., Alba, E., and Luna, F. (2011c). Elementary landscape decomposition of the frequency assignment problem. Theoretical Computer Science, 412(43):6002 - 6019 .

Deb, K., Pratap, A., Agarwal, S., and Meyarivan, T. (2002). A fast and elitist multiobjective genetic algorithm: NSGA-II. IEEE Transactions on Evolutionary Computation, 6(2):182-197.

Drezner, Z., Hahn, P., and Taillard, É. (2005). Recent advances for the quadratic assignment problem with special emphasis on instances that are difficult for meta-heuristic methods. Annals of Operations Research, 139(1):65-94.

Finner, H. (1999). Stepwise multiple test procedures and control of directional errors. Ann. Statist., 27(1):274-289.

Glover, F., Alidaee, B., Rego, C., and Kochenberger, G. (2002). One-pass heuristics for large-scale unconstrained binary quadratic problems. European Journal of Operational Research, 137(2):272 - 287. Graphs and Scheduling. 
Glover, F., Kochenberger, G. A., and Alidaee, B. (1998). Adaptive memory tabu search for binary quadratic programs. Management Science, 44(3):336-345.

Good, P. (2013). Permutation tests: a practical guide to resampling methods for testing hypotheses. Springer Science \& Business Media.

Grover, L. K. (1992). Local search and the local structure of NP-Complete problems. Operations Research Letters, 12(4):235 - 243.

Handl, J., Lovell, S., and Knowles, J. (2008). Multiobjectivization by decomposition of scalar cost functions. In Rudolph, G., Jansen, T., Lucas, S., Poloni, C., and Beume, N., editors, Parallel Problem Solving from Nature - PPSN X, volume 5199 of Lecture Notes in Computer Science, pages 31-40. Springer Berlin Heidelberg.

Knowles, J., Watson, R., and Corne, D. (2001). Reducing local optima in single-objective problems by multi-objectivization. In Zitzler, E., Thiele, L., Deb, K., Coello Coello, C., and Corne, D., editors, Evolutionary Multi-Criterion Optimization, volume 1993 of Lecture Notes in Computer Science, pages 269-283. Springer Berlin Heidelberg.

Koopmans, T. C. and Beckmann, M. J. (1955). Assignment Problems and the Location of Economic Activities. Cowles Foundation Discussion Papers 4, Cowles Foundation for Research in Economics, Yale University.

Lim, M. H., Yuan, Y., and Omatu, S. (2000). Efficient genetic algorithms using simple genes exchange localsearch policy for the quadratic assignment problem. Computational Optimization and Applications, 15(3):249-268.

Martí, R. and Reinelt, G. (2011). The linear ordering problem: exact and heuristic methods in combinatorial optimization, volume 175 . Springer.

Neumann, F. and Wegener, I. (2006). Minimum spanning trees made easier via multiobjective optimization. Natural Computing, 5(3):305-319.

Rana, S., Heckendorn, R. B., and Whitley, D. (1998). A tractable walsh analysis of SAT and its implications for genetic algorithms. In In Proceedings of AAAI, pages 392-397.

Rockmore, D., Kostelec, P., Hordijk, W., and Stadler, P. (2002). Fast fourier transforms for fitness landscapes. Applied and Computational Harmonic Analysis, 12(1):57-76.

Scharnow, J., Tinnefeld, K., and Wegener, I. (2005). The analysis of evolutionary algorithms on sorting and shortest paths problems. Journal of Mathematical Modelling and Algorithms, 3(4):349-366.

Segura, C., Coello, C. A. C., Miranda, G., and Leon, C. (2013). Using multi-objective evolutionary algorithms for single-objective optimization. 4OR, 11(3):201-228.

Stadler, P. F. (1996). Landscapes and their correlation functions. Journal of Mathematical Chemistry, 20:1-45.

Stadler, P. F. (2002). Fitness landscapes. Applied Mathematics and Computation, 117:187207.

Sutton, A. M., Whitley, L. D., and Howe, A. E. (2009). A polynomial time computation of the exact correlation structure of k-satisfiability landscapes. In Proceedings of the 11th Annual Conference on Genetic and Evolutionary Computation, GECCO '09, pages 365-372, New York, NY, USA. ACM. 
J. Ceberio, B. Calvo, A. Mendiburu and J. A. Lozano

Whitley, D., Sutton, A. M., and Howe, A. E. (2008). Understanding elementary landscapes. In Proceedings of the 10th Conference on Genetic and Evolutionary Computation, GECCO '08, pages 585-592, New York, NY, USA. ACM.

Zhang, Q. and Li, H. (2007). MOEA/D: a multiobjective evolutionary algorithm based on decomposition. IEEE Transactions on Evolutionary Computation, 11(6):712-731.

Zitzler, E., Laumanns, M., and Thiele, L. (2001). SPEA2: Improving the Strength Pareto Evolutionary Algorithm for Multiobjective Optimization. In Giannakoglou, K. et al., editors, Evolutionary Methods for Design, Optimisation and Control with Application to Industrial Problems (EUROGEN 2001), pages 95-100. International Center for Numerical Methods in Engineering (CIMNE). 